\title{
Naturerkenntnis und Dichtung
}

\author{
Von Heinz Balmer
}

\section{Die Gefahr der Zeitwidrigkeit}

Die Naturerkenntnis dringt in die Dichtung ein. Zwar braucht nach Lessing das Genie «tausend Dinge nicht zu wissen, die jeder Schulknabe weiß» - weil es beim Künstler auf das Gefühl ankommt ${ }^{1}$. Das historische Drama aber darf seine Gestalten nicht mit späteren Errungenschaften ausstatten. Goethe ließ ursprünglich Götzens Frau Elisabeth im ersten Aufzug sagen, sie sei «mit Kartoffeln und Rüben erzogen». Als er wahrnahm, daß die Kartoffel um 1500 noch nicht eingeführt war, strich er die Zeile ${ }^{2}$. So frei er die Personen formte, so fest hielt er sich an die Umstände.

Shakespeare lebte so sehr in einer Zeit knallender Pistolen und Kanonen, daß er verga $\beta$, daß die Feuerwaffe mit Schießpulver erst im 14 . Jahrhundert aufgekommen war. Im «Perikles» (I, 1) droht der Antiochier Thaliard: «Wenn ich ihn in Reichweite meiner Pistole bekomme» (If I can get him within my pistol's length), und im «Hamlet» $(I, 2)$ verfügt König Claudius (zu Anfang des 13. Jahrhunderts), das Geschütz solle «heut jeden frohen Trunk, Den Dänmark ausbringt, an die Wolken tragen» (the great cannon to the clouds shall tell).

In der Aufklärungszeit durchdrangen die Fortschritte der Physik derart die Geister ${ }^{3}$, daß man sich bewußt über Raum und Zeit hinwegsetzte. Johann Jakob Bodmer malte 1752 im Epos «Der Noah» in Hexametern die Sintflut aus. Ein Komet saugt das Meerwasser empor und läßt es gießen. Die Familie Noahs, die in einem gartengleichen Alpental gewohnt hat, besteigt die Arche. Darin betrachten die Geretteten die Landschaften auf den gemalten englischen Tapeten. Mit der Lupe belehren sie sich über die Augen der Fliege. Bedeutende Zeitgenossen verglichen Bodmer mit Homer. Herder dagegen tadelte, daß der Okzident in den Orient und das 18. Jahrhundert vor die Sintflut übertragen seien. Bodmer habe «die Tafeln aller Zeiten geplündert». ${ }^{4}$

Die Elektrizitätsforschung hatte 1600 mit William Gilbert begonnen, der geriebene Gegenstände auf ihre Anziehung prüfte und das Wort «elektrisch» vom Bernstein auf andere Stoffe übertrug. Jedoch erst die Elektrisiermaschine und der Blitzableiter machten die Elektrizität nach 1750 volkstümlich. Otto von Guericke ging 1663 mit der drehbaren Schwefelkugel voraus; Francis Hawksbee beobachtete 1706 bei der geriebenen Glaskugel Funken. 
Zwischen 1740 und 1750 wurde die Maschine mit Kurbel, Konduktor und Reibzeug ergänzt. Je deutlicher sie knisterte und sprühte, um so mehr dachte man an Blitz und Donner. Franklins Drache stieg in die Gewitterwolke und erwies die darin vermutete Elektrizität. 1752 erkannte LouisGuillaume Le Monnier, daß die Atmosphäre auch sonst elektrisch ist ${ }^{5}$.

Wenn Conrad Ferdinand Meyer im 7. Kapitel der Novelle «Das Amulett» Montaigne am 23. August 1572 zum Rat Chatillon sprechen läßt: «Das kann die Atmosphäre schon elektrisch machen», so ist dieser Wortlaut genau 180 Jahre verfrüht. Auch Wallenstein, der 1583-1634 lebte, hat den Blitzableiter nicht gekannt. Trotzdem rühmt der Dragoneroberst Buttler Schillers Wallenstein:

«Und wie des Blitzes Funke sicher, schnell,

Geleitet an der Wetterstange, läuft,

Herrscht sein Befehl vom letzten fernen Posten.» ${ }^{6}$

Ein anderes eindrucksvolles Zeitereignis der Aufklärung war 1782 der Luftballon der Brüder Montgolfier. Ein Strohfeuer an der offenen Grundfläche der Hülle verlieh ihrer Innenluft Steigkraft. Kühn setzte Goethe der Montgolfiere gleichsam eine zeitlose Mephistopheliere zur Seite, denn sein Mephisto will (um 1510) mit Faust nach Auerbachs Keller fliegen:

«Ein bißchen Feuerluft, die ich bereiten werde,

Hebt uns behend von dieser Erde.

Und sind wir leicht, so geht es schnell hinauf.»

\section{Schiller und Scheuchzer}

In Schillers Schauspiel «Wilhelm Tell» fallen die vielen Stellen zur Naturgeschichte der Alpenwelt auf. Die Angaben über Gewitteranzeichen, Föhn, Mondregenbogen, Gemsjagd, Gletscher, Lawinen, Hagel und Bergsturz, ebenso Ortsnamen gehen zurück auf Schriften des Zürcher Naturforschers Johann Jakob Scheuchzer, nämlich: «Seltsamer Naturgeschichten Des Schweizer-Lands Wochentliche Erzehlung» (3 Bände, Zürich 1705-1707) und «Natur-Histori des Schweitzerlands» (3 Bände, Zürich 1716-1718). Das erste Werk erschien ursprünglich als Wochenblatt mit lockerer Themenfolge; das zweite bot Übersicht und Ergänzungen in straffer Gliederung ? Im folgenden sind sie als «Wochenschrift» und «Natur-Histori» auseinandergehalten. Nach Schillers Hauptquelle, Aegidius Tschudis «Chronicon Helveticum», geschah Tells Tat am 19. November 1307. Den Rütlischwur verbinden wir heute mit dem Bundesbrief vom August 1291. Unbedenklich hat 
Schiller die Gründer der Eidgenossenschaft mit Scheuchzers Naturkunde ausgestattet.

Wilhelm Tell botanisierte und sammelte Fossilien. Wir erfahren es aus seinem Selbstgespräch in der hohlen Gasse (IV, 3). Niemals sei er ohne schöne Alpenblume oder Ammonshorn heimgekehrt. Jetzt gehe er einem andern Weidwerk nach und sitze am wilden Weg mit Mordgedanken.

Vom Cornu Ammonis oder Ammons-Horn berichtet Scheuchzer ausführlich in seiner Natur-Histori (Bd.3, S. 252-274). Allerdings nennt er fast nur Fundstellen im Juragebiet. Schiller ließ sich wohl von der Angabe verleiten, das Ammonshorn sei sehr verbreitet (S.253). «Wann wir Schweitzer aller Orthen, wo dergleichen Cornua Ammonis gefunden wurden, müßten dem Jupiter zu Gefallen Tempel und Götzen-Bilder aufrichten, so müßten wir auf vielen Höhen räucheren.»

Es ist reizvoll, wie Schiller Angaben Scheuchzers in Verse umgegossen hat. Das Schauspiel beginnt mit einem nahenden Gewitter, Scheuchzers Wochenschrift mit einer Zusammenstellung «Von den Vorbotten des Regens» (S.9-13). Gemeiniglich erfolge ein Regen, «wann die Enten, und andere W asservögel sich oft eintunken». Auch weitere Tiere zeigen bevorstehenden Regen an: «die Schaaffe mit begirriger auffressung des Grases ... der Hund mit aufscharrung der Erde ... Die stummen Fische ... durch ungewohnte Sprünge ausser das Wasser.» Die sichersten Anzeichen aber seien Wolken, «wann die sich in die tieffe Luft herab lassen, und an den Bergen umher kleben». In Engelberg pflege man zu sagen, wenn Wolken in das Tal einmarschieren: «der graue Thalvogt komt». Ein Bündner Berg bei Filisur habe vor dem Regen «eine Kappe auf». Schließlich halten «die Alpler» es für ein sicheres Zeichen einfallenden Regens, «wann der Firn, oder das beständige Berg-Eis brület».

Bei Schiller $(I, 1)$ sprechen am Gestade gegenüber Schwyz der Fischer Ruodi, der Hirt Kuoni und der Jäger Werni miteinander. Die Vorstellungsgabe des Dichters hat den Ausspruch vom Talvogt und vom Firn dorthin verpflanzt, die Kappe den Mythen umgehängt und in den Urnersee ein Wasserhuhn gesetzt.

$\begin{array}{ll}\text { Ruodi: } & \text { «Der graue Talvogt kommt, dumpf brült der Firn, } \\ & \text { Der Mythenstein zieht seine Haube an ...» } \\ \text { Kuoni: } & \text { «'s kommt Regen, Fährmann. Meine Schafe fressen } \\ & \text { Mit Begierde Gras, und Wächter scharrt die Erde.» } \\ \text { Werni: } & \text { «Die Fische springen, und das Wasserhuhn } \\ & \text { Taucht unter. Ein Gewitter ist im Anzug.» }\end{array}$


Scheuchzer erzählt von dem starken Föhnwind, der zu Ausgang des Oktobers 1705 die schweizerischen Lande durchweht habe ${ }^{8}$. Bei Schiller wird daraus der Tag der Heiligen Simon und Judas (28. Oktober):

Ruodi: «'s ist heut Simons und Judä, Da rast der See und will sein Opfer haben.»

Tell $(I, 3)$ braucht den Föhn als Vergleich:

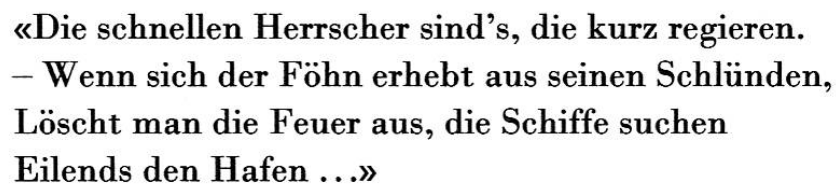

Diese Rede folgt Scheuchzers Worten, wonach man besonders seit dem Brand in Altdorf 1693 «kein Feuer anzuzünden gewahrnet wird» und «sich dannzumahl niemand darff auf den See wagen». ${ }^{9}$

Das Wochenblatt berichtet von einem Mondregenbogen, der 1705 «den 31 . Octob. Abends von $7 \frac{1}{2}$ bis nach 9 Uhren» von Schwyz gegen Unterwalden über dem See erblickt worden sei ${ }^{10}$. Eine Abbildung zeigt ihn. Das Licht des tief stehenden Vollmondes bei ruhiger Luft sei dazu nötig, die Erscheinung selten, «so daß wir innert 200 Jahren nur 5 bis 6 wahre Monds Regenbögen haben». Am Vierwaldstättersee stand über dem ersten Bogen «noch ein anderer, secundaria, wiewol der nicht die völlige Rundung hatte, wie der erste, auch gar bleich von Farben gewesen».

Schiller überträgt den Mondregenbogen von 1705 auf die Rütli-Szene (II, 2). Während die Unterwaldner auf die Ankunft der Schwyzer und Urner warten, führen sie ein physikalisches Gespräch in Scheuchzers Tonart:

\begin{tabular}{|c|c|}
\hline \multirow{3}{*}{$\begin{array}{l}\text { Von der Flüe: } \\
\text { Meier: }\end{array}$} & 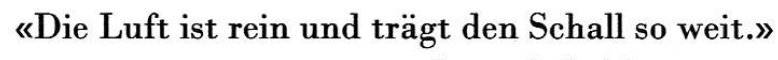 \\
\hline & \\
\hline & Ein $\mathbf{F}$ \\
\hline & «Es ist das Licht \\
\hline on d & Zeichen! \\
\hline & Es l \\
\hline & lt; seht, ein blässerer st \\
\hline
\end{tabular}

Nach der Szene mit dem Apfelschuß (III, 3) wird Tell auf Geßlers Schiff über den Urnersee abgeführt. Es hagelt und stürmt. Ein Fischer am Ufer (IV, 1) würde sich vor Empörung nicht wundern, wenn die Eistürme «Von ihren hohen Kulmen niederschmelzen». Das ungewöhnliche Wort für Gipfel 
stammt aus Scheuchzers Wochenschrift: «Die oberste Höhe des Rigibergs im Schweitzergebieth heisset Kulm.» ${ }^{11}$ Die Ortsangaben zum Schiff im Sturm (Buggisgrat, Teufelsmünster, großer Axenberg, Hackmesser, Kleiner Axen) sind aus Scheuchzers Karte des Urnersees übernommen ${ }^{12}$.

In seiner Wochenschrift beschreibt Scheuchzer die Gefahren der Gemsjagd $^{13}$. Das in die Enge getriebene Tier könne den Jäger anfallen und mit ihm über die Felswand abstürzen. Davon spricht Tells Frau (III, 1). Oder der Jäger könne sich derart versteigen, daß nur ein «Wagsprung» ihn rette. Um dabei nicht auszugleiten, ziehe er die Schuhe aus und schneide sich «mit dem Messer in die Fersen, oder Ballen des Fusses», damit das hervorwallende Geblüt ihn «an statt eines Leims» am Felsen fest anhalte. Im Selbstgespräch in der hohlen Gasse (IV, 3) denkt Tell an diesen «Wagesprung» des Jägers an den glatten Wänden, «Wo er sich anleimt mit dem eignen Blut, Um ein armselig Grattier zu erjagen». Dieses Anleimen mit Fußblut wurde in unserem Jahrhundert als Wahrheit bestätigt ${ }^{14}$.

Bevor Geßler naht, begegnet der Flurschütz Stüssi dem wartenden Tell.

Stüssi:

«Es gibt allerwegen

Unglücks genug. Ein Ruffi ist gegangen

Im Glarner Land, und eine ganze Seite

Vom Glärnisch eingesunken.»

Tell:

«Wanken auch

Die Berge selbst? Es steht nichts fest auf Erden.»

In der Natur-Histori erklärt Scheuchzer (Bd.1, S.128): «Bergfälle, Bergschlipfen, Bergbrüche nennen wir, wann ein ganzer Berg, oder ein Theil desselben ein- oder von der Höhe hinunterfallet: hin und wieder nennet man sie Rüfen, Rüfinen, vielleicht à rupibus, von Felsen, welche bey dergleichen Anläsen in die Thäler hinunter fallen.» Unter den Beispielen erwähnt er (S. 134), daß «1593 an S. Martins Abend» (am 11. November) «ein groß stuck Felsen von dem Glärnischberg abgeschrenzt, und mit einem entsetzlichen Braschlen auf die Seiten gegen dem Hauptflecken Glarus hinunter gerollet». 1594 folgten am Glärnisch weitere Felsabbrüche. Den Ausdruck «ein Rufi gangen» findet man auf Seite 136 oben.

In der vorletzten Szene (V, 2) beschreibt Tell dem flüchtenden Parricida den Weg zur Gotthardhöhe über «die Brücke, welche stäubet».

«Wenn Ihr sie glücklich hinter Euch gelassen,

So reißt ein schwarzes Felsentor sich auf-

Kein Tag hat's noch erhellt -, da geht Ihr durch.» 
Gemeint ist das Urnerloch ${ }^{2}$. Es schloß sich nicht an die stiebende Brücke an, sondern ersetzte sie. Erst 1707-1708 wurde es von Pietro Morettini aus Cerentino erbaut.

Daß ein Föhnsturm und ein Mondregenbogen von Ende Oktober 1705 und ein Glärnisch-Bergsturz von 1593 in das Drama eingezogen sind, stört nicht, weil diese Naturereignisse überzeitlich sind. Der Dichter verwandelt die Nachrichten in Sinnbilder, die nicht nur Lokalfarbe geben, sondern Stimmungen tragen. Gefährlich wird Scheuchzers Einfluß nur dort, wo Schiller die Urschweizer mit der Naturauffassung der Aufklärung anhaucht, indem er Tell Ammoniten heimbringen oder Melchthal den Regenbogen erläutern läßt.

Um so eindrücklicher erkennt man die Kraft des Dichters, sobald eine Beobachtung nur als Gesimse dient, von dem aus der Geist sich wie ein Vogel zu Gefilden ewiger Wahrheit emporschwingt. Wenn Scheuchzer im ersten Band der Wochenschrift das Heimweh der Schweizer schildert, deutet er es aus dem höheren Luftdruck über fremden Ländern und umwickelt die Kunde mit Erklärungen. In Schiller aber erwachen Gedanken über den Trieb des Vaterlands (II,1). Sie münden in die Worte Attinghausens:

«Ans Vaterland, ans teure, schließ dich an,

Das halte fest mit deinem ganzen Herzen!»

\section{Quellenhinweise}

${ }^{1}$ Lessing am Beginn des 34. Stückes der Hamburgischen Dramaturgie.

${ }^{2}$ Hanns Braun, Hier irrt Goethe, München 1937; letzte Bearbeitung als dtv-Taschenbuch, München 1966.

3 Vgl. Andreas Kleinert, Die allgemeinverständlichen Physikbücher der französischen Aufklärung (Veröffentlichungen der Schweizerischen Gesellschaft für Geschichte der Medizin und der Naturwissenschaften 28), Aarau 1974.

4 Johann Ulrich Marbach, Herder und die schweizerische Literatur, Diss. Bern, Neapel 1954, S. 191-209.

5 Ludwig Darmstaedter, Handbuch zur Geschichte der Naturwissenschaften und der Technik, 2. Auflage, Berlin 1908.

6 Die Piccolomini I, 2.

7 Von Scheuchzers drei Bänden der Natur-Histori des Schweitzerlands hat Arthur Dürst im Orell Füßli Verlag Zürich 1978-1979 eine Faksimileausgabe mit trefflichem Begleittext (in einer Tasche hinten in Bd.1) veranstaltet.

${ }^{8}$ Wochenschrift ( = Seltsamer Naturgeschichten Des Schweizer-Lands Wochentliche Erzehlung), Bd.1, S. 181.

9 Wochenschrift, Bd. 3, S. 38. 
${ }^{10}$ Wochenschrift, Bd.2, S.43. Vgl. Erwin Bührer, Quellen, die Schiller zu seinem Wilhelm Tell benützte. In: Die neue Schulpraxis 21 (Heft 7, Juli 1951), S.257-259 (mit 2 Abbildungen).

11 Wochenschrift, Bd.1, S. 106.

12 Natur-Histori, Bd. 1, bei S.112. Vgl. wiederum Erwin Bührer (Anm. 10).

${ }^{13}$ Wochenschrift, Bd.1, S.42 und 43.

${ }^{14}$ Hans Rhyn, Schweizer Wilderergeschichten, Aarau 1923, Anmerkung S. 53 (2. Auflage: Gute Schriften, Bern 1963, S.47).

\section{Summary}

Every author of a historical drama or novel, even when he is well informed in the history of science, is in danger to anticipate some invention or knowledge. There are discussed sayings in works of Shakespeare, Goethe, Schiller, and C.F.Meyer, concerning the gun-powder, the airballoon, the lightning-rod, and atmospheric electricity. The second part deals with Schiller's William Tell and shows the influence of Scheuchzer's Natural History of Switzerland on it. Schiller found there the special alpine atmosphere. He transfered not only the description of phenomena, but also the feeling for nature from 1700 to 1300 . His poetic inspiration proved successful.

Dr. Heinz Balmer

Medizinhistorisches Institut

Rämistraße 71

CH-8006 Zürich 